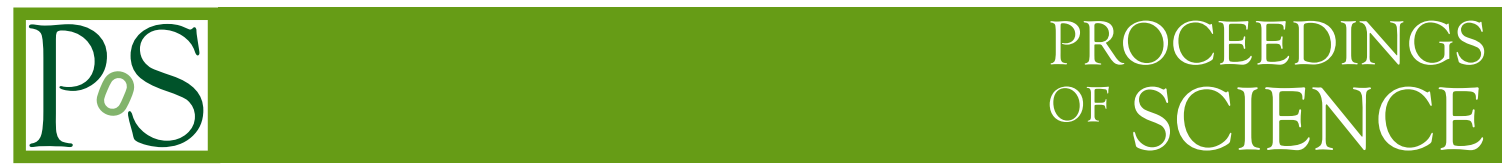

\title{
Strongly coupled plasma - hydrodynamics, thermalization and nonequilibrium behavior
}

\author{
Romuald JANIK* \\ Jagiellonian University, Kraków \\ E-mail: romualdeth.if.uj.edu.pl
}

In this talk I will describe various features of time-dependent dynamics of strongly coupled plasma from the perspective of the AdS/CFT correspondence. I will take as an example boostinvariant plasma flow and concentrate on the properties of hydrodynamic expansion, thermalization versus hydrodynamization and some features of nonequilibrium behaviour.

KMI International Symposium 2013 on "Quest for the Origin of Particles and the Universe" 11-13 December, 2013

Nagoya University, Japan

\footnotetext{
* Speaker.
} 


\section{Introduction and motivation}

One of the outstanding theoretical problems, relevant for the experimental heavy-ion programs at LHC and RHIC, is the understanding of real-time dynamics of far-from-equilibrium quark-gluon plasma. This physics is especially elusive, as the quintessential tool of nonperturbative QCD lattice QCD cannot be applied, since it is formulated in Euclidean signature.

In this talk I will concentrate on an approach to these kind of problems through the AdS/CFT correspondence [1], which postulates an equivalence between $\mathscr{N}=4$ supersymetric gauge theory and superstring theory in $A d S_{5} \times S^{5}$ spacetime. The reasons which make this correspondence an effective tool for studying the relevant physics are twofold. It is formulated directly in Minkowski signature and moreover, at strong coupling, the dual description essentially reduces to classical gravity for which we have effective calculational tools. Of course, the downside is that we are no longer dealing directly with QCD but rather with a different gauge theory. Fortunately, once we are studying plasma physics (i.e. QCD physics for temperatures above the confinement-deconfinement phase transition/crossover), the chief differences from QCD go away. In particular, supersymmetry is explicitly broken by the temperature, which also becomes the dominant scale. Indeed, recent investigation of transport and kinetic equations in $\mathscr{N}=4 \mathrm{SYM}$ indicate that the differences with analogous computations in QCD amount essentially just to a difference in the number of degrees of freedom [2].

However, having said all this, one has to nevertheless keep in mind some differences which may be relevant for various concrete questions. One key difference is the absence of a phase transition in $\mathscr{N}=4 \mathrm{SYM}$. Consequently, the plasma expansion and cooling will continue indefinitely. Secondly, the coupling in $\mathscr{N}=4 \mathrm{SYM}$ is not running and has to be large in order for the AdS/CFT methods to be effective in the plasma context. So in particular mixed soft/hard physics may be significantly different. Therefore, the (qualitative) relevance of particular computations in $\mathscr{N}=4$ SYM for QCD problems has to be judged depending on the concrete physical question considered. Moreover, one should eventually consider passing to more complicated AdS/CFT setups which involve gauge theories which might be closer to QCD.

Before I finish this brief introduction, I would like to emphasize that the AdS/CFT correspondence provides for us a concrete example of a strongly coupled gauge theory in which we may study in very much detail nonperturbative real-time dynamics. We may then confront our theoretical expectations and prejudices with these results and investigate possible relevance for realistic quark-gluon-plasma dynamics. One may also hope to obtain certain quantitative answers which may serve as a useful reference point for QCD applications - the key example is the famous shear viscosity to entropy ratio $[3,4]$.

\section{Boost invariant flow}

In this talk I will review our work on exploring the interrelations between hydrodynamic and nonequilibrium behaviour within the context of a boost-invariant plasma configuration. Such a setup is a very appealing laboratory for exploring nonequilibrium physics since the symmetry assumptions significantly reduce the complexity of the problem. Then the dual AdS gravitational description is essentially two-dimensional, which simplifies the numerical solution of Einstein's 
equations. At the same time, the assumed symmetry is not overconstraining the system and almost all of the physics relevant for thermalization is still present. Indeed, we may and do observe complicated nonequilibrium dynamics at early times and a gradual transition to hydrodynamic evolution.

These symmetry assumptions were first proposed by Bjorken in [5] as an approximate description of a plasma fluid produced in a a very highly energetic collision. Thus, following Bjorken, we assume invariance under boosts in the longitudinal plane. Moreover, we assume unifomity in the transverse directions. With these assumptions in place, the conservation of energy-momentum and the equation of state (which for the $\mathscr{N}=4$ theory is just the tracelessness condition of $T_{\mu v}$ ) implies that all components of $T_{\mu \nu}$ can be expressed in terms of just a single function $\varepsilon(\tau)$, which is the energy density at mid-rapidity. In particular, the longitudinal and transverse pressures (defined through the appropriate diagonal components of $T_{\mu \nu}$ ) are given by

$$
p_{L}=-\varepsilon-\tau \frac{d}{d \tau} \varepsilon \quad p_{T}=\varepsilon+\frac{1}{2} \tau \frac{d}{d \tau} \varepsilon
$$

In the following, it turns to be convenient to use the notion of effective temperature $T_{\text {eff }}$ which is defined to be the temperature of a thermal system in $\mathscr{N}=4$ SYM with energy density equal to the instantenous energy density $\varepsilon(\tau)$ :

$$
\varepsilon(\tau)=\frac{3}{8} N_{c}^{2} \pi^{2} T_{e f f}^{4}(\tau)
$$

In this way one can get rid of the trivial differences between gauge theories due to various numbers of degrees of freedom.

\section{Hydrodynamics in the boost invariant setting}

Let us now revisit hydrodynamics in the boost invariant setting. Hydrodynamics amounts to the assumption that the whole long wavelength dynamics is completely expressible in terms of a local flow velocity and local temperature ${ }^{1}$. Then the energy-momentum tensor $T_{\mu \nu}$ is expressed in an expansion in the number of derivatives of the flow velocity.

$$
T_{\text {hydro }}^{\mu v}=\frac{1}{3} \varepsilon\left(4 u^{\mu} u^{v}+\eta^{\mu v}\right)+\eta\left(P^{\mu \alpha} P^{v \beta} \partial_{(\alpha} u_{\beta)}-\frac{1}{3} P^{\mu v} \partial_{\alpha} u^{\alpha}\right)+\ldots
$$

The leading term corresponds to a perfect fluid, the $1^{\text {st }}$ correction is just the shear viscosity multiplying the standard shear tensor and subsequent terms would involve new, higher order transport coefficients and new tensorial structures with more than one derivative of the flow velocity $u^{\mu}$ (see $[6,7]$ for explicit expressions). Hydrodynamic equations of motion are first order equations given just by energy-momentum conservation ${ }^{2}$

$$
\partial_{v} T_{h y d r o}^{\mu v}=0
$$

The expansion (3.1) can, in fact, be rigorously derived from AdS/CFT [7]. This procedure is usually called fluid/gravity duality, although this name may be slightly misleading as gravity within AdS/CFT contains a much richer range of dynamics than just (generalized) fluid dynamics.

\footnotetext{
${ }^{1}$ Here I assume the conformal equation of state $E=3 p\left(T_{\mu}^{\mu}=0\right)$.

${ }^{2} \mathrm{I}$ am not discussing here the Israel-Stewart formulation.
} 
In the boost invariant setting (with no transverse dependence), the flow velocity is completely fixed by symmetry and the only degree of freedom is the dependence of the temperature on the (longitudinal) proper time $T(\tau)$. Let us note that this $T(\tau)$ would coincide with our definition of effective temperature $T_{\text {eff }}$ in the previous section. The hydrodynamic equations of motion become just a $1^{s t}$ order (nonlinear) ODE for $T(\tau)$.

These simple facts have significant consequences. They imply that once $T(\tau)$ has some fixed value at a given time, the rest of the evolution of $T(\tau)$ is completely fixed ${ }^{3}$ and unique. This conclusion holds irrespective of any precise knowledge about the higher order hydrodynamic terms in the derivative expansion (3.1).

Alternatively, once we fix the late-time asymptotics of $T(\tau)$ through

$$
\pi T(\tau) \sim \frac{1}{\tau^{\frac{1}{3}}}
$$

with a given fixed coefficient in the numerator, the hydrodynamic evolution of a plasma system would be a unique fixed curve $T_{\text {hydro }}(\tau)$. Any deviations from this curve would be due to the presence other non-hydrodynamic excitations of the strongly coupled plasma system. In particular, one could imagine preparing the plasma system at $\tau=0$ in various ways, giving various initial conditions to these non-hydrodynamic degrees of freedom ${ }^{4}$. Then we would expect to observe a plethora of various curves $T_{\text {eff }}(\tau)$ which would converge to the single hydrodynamic curve $T_{\text {hydro }}(\tau)$.

Another interesting issue is what exactly is the shape of the hydrodynamic curve $T_{\text {hydro }}(\tau)$. The answer is of course well known if we choose to restrict ourselves just to $1^{\text {st }}$ order viscous hydrodynamics (or up to $3^{\text {rd }}$ order in the boost-invariant case). However, as we go to earlier and earlier times, dissipation becomes more and more important and higher order terms in the hydrodynamic derivative expansion in (3.1) become relevant. Within the AdS/CFT correspondence, we recently obtained numerically around 240 subsequent terms in the (boost-invariant) hydrodynamic expansion [9]. It turns out that the hydrodynamic series has zero radius of convergence and is only asymptotic. However, there are strong indications that it is Borel summable. Currently we are investigating approximate Borel resummations which may serve as a benchmark of resummed $T_{\text {hydro }}(\tau)$.

The time after which the relevant curve $T_{\text {eff }}(\tau)$ would approach $T_{\text {hydro }}(\tau)$ would be the hydrodynamization time - the time after which a purely hydrodynamic description of the plasma would be valid. Knowing the energy-momentum tensor, allows us to ask whether the diagonal components (i.e. longitudinal and transverse pressures) are approximately isotropic which would be a necessary condition for local thermal equillibrium.

One of the aims of the AdS/CFT investigations of the boost-invariant plasma system was to verify whether the above physical picture indeed holds, and to understand its fine details.

\section{The AdS/CFT description}

As the AdS/CFT correspondence is expected to be an equivalence between $\mathscr{N}=4 \mathrm{SYM}$ theory and superstring theory in $A d S_{5} \times S^{5}$, each gauge theory state/phenomenon should have a counterpart on the dual side. We thus seek to describe the time-dependent evolving strongly coupled

\footnotetext{
${ }^{3}$ In an Israel-Stewart formulation one may include one other initial condition.

${ }^{4}$ Of course, all these degrees of freedom are expected to interact nonlinearly between themselves.
} 
plasma system in terms of the dual degrees of freedom on the string side of the correspondence. At strong (gauge theory) coupling, there appears a huge mass gap between gravity modes in $A d S_{5} \times S^{5}$ and degrees of freedom corresponding to massive string states. Hence one may expect to describe the evolving plasma system purely within gravity ${ }^{5}$. A proposal for doing this was formulated in [10]. Some additional assumptions amounting to not turning on any exotic charges in $\mathscr{N}=4$ SYM, allow us to reduce the description to a 5D geometry:

$$
d s^{2}=\frac{g_{\mu v}\left(x^{\rho}, z\right) d x^{\mu} d x^{v}+d z^{2}}{z^{2}} \equiv g_{\alpha \beta}^{5 D} d x^{\alpha} d x^{\beta}
$$

where $z \geq 0$ is the $5^{\text {th }}$ coordinate, with the $A d S$ boundary given by $z=0$. Its time evolution is given by $5 \mathrm{D}$ vacuum Einstein's equations with negative cosmological constant:

$$
R_{\alpha \beta}-\frac{1}{2} g_{\alpha \beta}^{5 D} R-6 g_{\alpha \beta}^{5 D}=0
$$

Given the $5 \mathrm{D}$ geometry $g_{\alpha \beta}^{5 D}$, one may extract the spacetime profile of the gauge theory energy momentum tensor $T_{\mu \nu}[11]$

$$
g_{\mu v}\left(x^{\rho}, z\right)=\eta_{\mu v}+g_{\mu v}^{(4)}\left(x^{\rho}\right) z^{4}+\mathscr{O}\left(z^{6}\right) \quad\left\langle T_{\mu v}\left(x^{\rho}\right)\right\rangle=\frac{N_{c}^{2}}{2 \pi^{2}} g_{\mu v}^{(4)}\left(x^{\rho}\right)
$$

Let us comment on the possible initial conditions and their interpretation. On the gravity side, the initial conditions are encoded in a choice of some specific geometry on a spacelike slice with $\tau=0$. Clearly we have an infinite dimensional space of possible initial conditions, even if we fix the initial energy density to a given value. This huge freedom of posing initial conditions is in marked contrast to hydrodynamics, where just the energy density ${ }^{6}$ at some given initial time would suffice.

On the gauge theory side, however, such freedom is also very natural. If we were at weak coupling, we could imagine preparing the initial state with various possible momentum distributions of gluons, each initial state having the same energy density. Then we could let the system evolve and study the approach of the momentum distributions to a gaussian thermal profile. The strong coupling analog of this picture would be to start the numerical evolution from various initial geometries and look for a transition to an eventual hydrodynamic description. This has been done in $[12,13]$ starting from $\tau=0$ and using various quite general initial conditions ${ }^{7}$

\section{Results}

The results presented in refs. $[12,13]$ corresponded to initial conditions with a common fixed energy density at $\tau=0$. Although from the point of view of an initial value problem this choice was very natural, it is quite inconvenient from the point of view of realistic relativistic heavy-ion

\footnotetext{
${ }^{5}$ Let us note that this does not hold at weak coupling, where the massive string states are equally important as gravity. Hence we lack a dual description of weakly coupled plasma

${ }^{6}$ Recall that we are discussing the boost invariant context.

${ }^{7}$ Previous numerical work on boost-invariant plasma started from $\tau=\tau_{0}>0$ and did not, by design, satisfy energymomentum conservation in the very initial stage [8].
} 


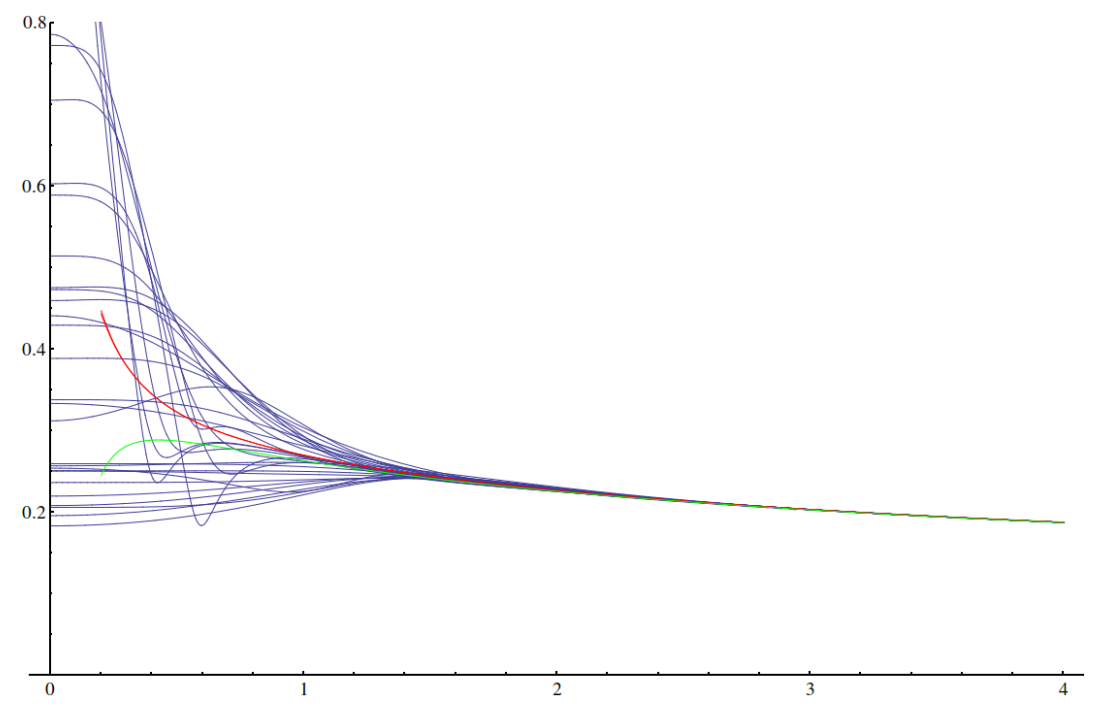

Figure 1: The effective temperature $T_{\text {eff }}$ as a function of $\tau$ for the various initial conditions considered in $[12,13]$. The green line represents $3^{\text {rd }}$ order hydrodynamic $T_{\text {hydro }}^{3^{\text {rd }} \text { order }}(\tau)$, while the red line corresponds to Borel resummed hydrodynamics $T_{\text {hydro }}^{\text {resummed }}(\tau)$ (colour online).

collisions, where we cannot really measure, even indirectly, the energy density at $\tau=0$. What is known is the temperature at late times (just before freezout) and final entropy/multiplicity. From hydrodynamic fits one then has a fairly good idea of the temperature up to the hydrodynamization time, but one cannot then proceed back to $\tau=0$ without some theoretical assumptions. Indeed, the AdS results reviewed below indicate that this cannot be done in a universal way.

For the above reasons it is interesting to recast the results of the numerical evolution keeping the large $\tau$ asymptotics (as in (3.3)) to be the same for all initial conditions. The plot of $T_{\text {eff }}(\tau)$ for the 29 initial conditions considered is shown in Fig. 1.

We see exactly the counterpart of the physical picture outlined in section 3. At late times all profiles follow very closely hydrodynamic evolution, however at small times we have a plethora of different curves corresponding to turning on various nonhydrodynamic degrees of freedom. Moreover, we see that knowing the temperature at the transition to hydrodynamics gives us no information about the temperature at $\tau=0$ unless we have some additional information about the initial state.

In order to discuss the finer details of the transition to hydrodynamics, it is convenient to introduce the dimensionless product $w=T_{e f f} \cdot \tau$. By construction it is independent of any overall scale as well as of the precise number of degrees of freedom in $\mathscr{N}=4$ SYM (since we are using the effective temperature in its definition).

One of the main findings of refs. $[12,13]$ was that even with a very stringent criterion of transition to hydrodynamics ${ }^{8}$, a hydrodynamic description holds for $w \geq 0.7$. Moreover for moderate and large initial data, $w$ at the transition to hydrodynamics is approximately constant ${ }^{9}$. It is interesting to compare this with sample initial data $T_{0}=500 \mathrm{MeV}, \tau_{0}=0.25 \mathrm{fm}$ used for RHIC in [14],

\footnotetext{
${ }^{8}$ I.e. that equations of motion of $3^{\text {rd }}$ order hydrodynamics are satisfied up to $0.5 \%$ accuracy.

${ }^{9}$ Here moderate and large initial data are distinguished by the value of their initial entropy (see below).
} 
which corresponds to $w=0.63$. The fact that these values are quite comparable suggests that even some quantitative intuition can be gained from studying strongly coupled $\mathscr{N}=4 \mathrm{SYM}$. Indeed, from the point of view of our numerical results, it is definitely not surprising that hydrodynamics should be applicable already at $T_{0}=500 \mathrm{MeV}, \tau_{0}=0.25 \mathrm{fm}$.

Another key feature which follows, is that at the transiton to hydrodynamics, the longitudinal and transverse pressures (diagonal components of $T_{\mu v}$ ) are quite different:

$$
\Delta p_{L} \equiv 1-\frac{p_{L}}{\varepsilon / 3} \sim 0.7
$$

This means that at the transition to hydrodynamics, the plasma is still quite far from local thermal equilibrium, but this deviation is completely explained by dissipative hydrodynamic flow. Thus hydrodynamization is distinct from thermalization and the 'early thermalization puzzle' appears to be really a misnomer.

Finally, we found that despite the varied dynamics apparent in the evolution profiles seen in Fig. 1, such key features of the evolution as entropy production or the hydrodynamization time are strongly correlated with just a single numerical characterization of the initial state - an initial entropy ${ }^{10}$.

Let me now describe some work in progress aiming at the understanding of the dynamics of the nonhydrodynamic degrees of freedom from a 4D perspective

\section{Beyond hydrodynamics - towards a four dimensional perspective}

As is clearly seen in Fig. 1, as well as in the very first numerical simulations in the boostinvariant context [8], the deviations from hydrodynamics occur through the appearance of new degrees of freedom in the plasma system. The appearance of a new degree of freedom is linked to the possibility of imposing independent initial conditions for this excitation (here we think directly in terms of the gauge theory in 4D). As can be intuitively seen in Fig. 1, more and more new effective degrees of freedom appear which reflects the infinite dimensional freedom of setting up initial conditions on the gravity side.

This infinite set of additional degrees of freedom can be, again intuitively, understood as being encoded in the additional dimension in the gravitational description. On the linearized level these degrees of freedom correspond to black hole quasi-normal modes which have a hierarchy of damping frequencies. Hence one may expect that in some intermediate region only a couple of these modes would be relevant. Preliminary analysis of our numerical evolution profiles compared to resummed hydrodynamics seem to support this conclusion.

A natural question which comes to mind is whether one can describe, perhaps approximately, the dynamics of such nonhydrodynamic degrees of freedom purely from a 4D perspective, in a way which agrees with what we know about the exact numerical description on the AdS side.

Let us recall that hydrodynamics amounts just to the assumption that the energy-momentum tensor $T_{\mu \nu}$ is completely expressible in terms of a scalar (temperature) and a unit vector (flow velocity) and an expansion in the number of derivatives. The description of additional nonhydrodynamic degrees of freedom would entail introducing additional independent effective fields into $T_{\mu \nu}$ and providing additional equations of motion. We hope to report on this issue in a future publication.

\footnotetext{
${ }^{10}$ Initial entropy is characterized geometrically through an area element of an apparent horizon.
} 


\section{Conclusions}

The AdS/CFT correspondence provides a very general framework for studying time-dependent dynamical evolution of strongly coupled plasma. Using these methods it is possible to follow the dynamics of the plasma system right from some far from equilibrium configuration through an initial out-of-equilibrium evolution (or even a collision process $[15,16]$ ) onto a very precise hydrodynamic description. These methods may thus give us qualitative insight into this very elusive nonperturbative real-time out-of-equilibrium regime of plasma dynamics. In some cases, once one factors out trivial differences due to the different number of degrees of freedom, one may even hope to get some semi-quantitative estimates. However, the key value of the AdS/CFT methods is that they provide for us a theoretical laboratory for testing various hypothesis and explicitly showing what kind of behaviour could be possible in a strongly coupled gauge theory - a key example of such an application is the observation that thermalization is distinct from hydrodynamization, namely that the onset of viscous hydrodynamics generically occurs when the pressures are still quite anisotropic.

\section{Acknowledgments}

This work was supported by NCN grant 2012/06/A/ST2/00396. I would like to thank the KobayashiMaskawa Institute for warm hospitality during the Symposium.

\section{References}

[1] J. M. Maldacena, "The Large N limit of superconformal field theories and supergravity," Adv. Theor. Math. Phys. 2 (1998) 231 [hep-th/9711200].

[2] A. Czajka and S. Mrowczynski, "N=4 Super Yang-Mills Plasma," Phys. Rev. D 86 (2012) 025017 [arXiv:1203.1856 [hep-th]].

[3] G. Policastro, D. T. Son and A. O. Starinets, "The Shear viscosity of strongly coupled N=4 supersymmetric Yang-Mills plasma,” Phys. Rev. Lett. 87 (2001) 081601 [hep-th/0104066].

[4] P. Kovtun, D. T. Son and A. O. Starinets, Phys. Rev. Lett. 94 (2005) 111601 [hep-th/0405231].

[5] J. D. Bjorken, "Highly Relativistic Nucleus-Nucleus Collisions: The Central Rapidity Region," Phys. Rev. D 27, 140 (1983).

[6] R. Baier, P. Romatschke, D. T. Son, A. O. Starinets and M. A. Stephanov, "Relativistic viscous hydrodynamics, conformal invariance, and holography," JHEP 0804 (2008) 100 [arXiv:0712.2451 [hep-th]].

[7] S. Bhattacharyya, V. E. Hubeny, S. Minwalla and M. Rangamani, "Nonlinear Fluid Dynamics from Gravity," JHEP 0802 (2008) 045 [arXiv:0712.2456 [hep-th]].

[8] P. M. Chesler and L. G. Yaffe, "Boost invariant flow, black hole formation, and far-from-equilibrium dynamics in N = 4 supersymmetric Yang-Mills theory," Phys. Rev. D 82 (2010) 026006 [arXiv:0906.4426 [hep-th]].

[9] M. P. Heller, R. A. Janik, P. Witaszczyk, "On the character of hydrodynamic gradient expansion in gauge theory plasma," Phys. Rev. Lett. 110 (2013) 211602 [arXiv:1302.0697 [hep-th]]. 
[10] R. A. Janik and R. B. Peschanski, "Asymptotic perfect fluid dynamics as a consequence of Ads/CFT," Phys. Rev. D 73 (2006) 045013 [hep-th/0512162].

[11] S. de Haro, S. N. Solodukhin, K. Skenderis, "Holographic reconstruction of space-time and renormalization in the AdS / CFT correspondence," Commun. Math. Phys. 217, 595-622 (2001). [hep-th/0002230].

[12] M. P. Heller, R. A. Janik, P. Witaszczyk, "The characteristics of thermalization of boost-invariant plasma from holography,” Phys. Rev. Lett. 108 (2012) 201602 [arXiv:1103.3452 [hep-th]].

[13] M. P. Heller, R. A. Janik, P. Witaszczyk, "A numerical relativity approach to the initial value problem in asymptotically Anti-de Sitter spacetime for plasma thermalization - an ADM formulation," Phys. Rev. D 85 (2012) 126002 [arXiv:1203.0755 [hep-th]].

[14] W. Broniowski, M. Chojnacki, W. Florkowski and A. Kisiel, "Uniform Description of Soft Observables in Heavy-Ion Collisions at $\mathrm{s}(\mathrm{NN}) * *(1 / 2)=200 \mathrm{GeV}^{* * 2}$," Phys. Rev. Lett. 101 (2008) 022301 [arXiv:0801.4361 [nucl-th]].

[15] P. M. Chesler and L. G. Yaffe, "Holography and colliding gravitational shock waves in asymptotically $A d S_{5}$ spacetime,” Phys. Rev. Lett. 106 (2011) 021601 [arXiv:1011.3562 [hep-th]].

[16] J. Casalderrey-Solana, M. P. Heller, D. Mateos and W. van der Schee, "From full stopping to transparency in a holographic model of heavy ion collisions," Phys. Rev. Lett. 111 (2013) 181601 [arXiv:1305.4919 [hep-th]]. 